\title{
Migrantinnen als Touristinnen zwischen Bolivien und Spanien
}

\author{
Von Juliane Müller
}

\section{Einleitung}

Die Hoheit über territoriale Grenzen ist ein Symbol nationalstaatlicher Souveränität in Zeiten wirtschaftlicher Globalisierung und transnationaler Verflechtungen. Im Zuge der Anschläge vom 11. September 2001 und einem gewachsenen Migrationsdruck haben die westlichen Staaten ihre Visapolitiken und Grenzkontrollen weiter verschärft. Dabei ist und bleibt ungeklärt, wie sich der Migrationsdruck unter freizügigeren Bedingungen entwickeln würde, ob er nicht gar abnehmen könnte (Wihtol de Wenden 2000). Angesichts der gegenwärtigen Tendenz immer höher gesteckter Mobilitätsbarrieren für Menschen aus den Ländern des Südens werden allerdings alle vorhandenen Möglichkeiten ausgelotet und Routen am Rande der Legalität genutzt, um nach Europa zu gelangen und nach Arbeit zu suchen.

Die jüngste südamerikanische Einwanderung nach Spanien, insbesondere aus den Andenländern, veranschaulicht eine der wenigen noch verbliebenen Chancen, ohne Visum in die Europäische Union zu reisen. Vor der Einführung des Schengen-Kurzzeitvisums für ihre Herkunftsländer bis 2007 benötigten die Migranten zwar einen Pass, Bargeld, ein Rückflugticket und andere kostspielige Garantien ihrer vermeintlichen Rückkehr, aber sie mussten keine weiteren Dokumente wie Geburtsurkunden, Arbeitsverträge oder Wohneigentum im Herkunftsland nachweisen; Visabedingungen, die die internationale Mobilität aus Entwicklungs- und Schwellenländern erheblich erschweren, wenn nicht unmöglich machen, und die Menschen auf gefährliche Geheimrouten ausweichen lassen. Außerdem wurden die Ausweispapiere und Reiseintentionen der Südamerikaner erst am Ankunftsflughafen kontrolliert, sodass sie sich auf den Weg machen konnten, um in Europa Arbeit zu suchen.

Wie aber muss man sich die Einreise der Migranten konkret vorstellen? Stießen sie trotz hinreichender Identitätsnachweise und Reisedo- 
kumente auf Probleme? Welche Taktiken nutzten sie bei der Unterredung mit Grenzbeamten? Welche Rolle spielte der Körper als physischer Marker ihrer ethnischen Herkunft?

Diese Fragen möchte ich am Beispiel bolivianischer Migrantinnen und ihrem Weg nach Spanien untersuchen. Es wird gezeigt, dass die Reise durch den ambivalenten Status zwischen Migrantin und Touristin geprägt wird, sowie erläutert, wie sich die Frauen selbst sehen und positionieren (vgl. Kopnina 2007: Kap. 3). Schließlich soll auch analysiert werden, wie nationale Politik, EU-Rechtsprechung und das Vorgehen von Reiseunternehmen bei den Arbeitswanderungen zwischen Bolivien und Spanien ineinandergreifen und was dies für die Migrantinnen bedeutet.

Konzeptionell wird davon ausgegangen, dass sich die Migrationsreise als ritueller Prozess begreifen lässt (Turner 1982). Die scharfen Kontrollen an den EU-Außengrenzen machen den Eintritt in den Schengenraum zum Schlüsselmoment für irreguläre Migranten aus Drittstaaten. Die Migrationsreise wird als eine biographische Übergangsphase interpretiert, die eine klare, auf Zeit angelegte physische Ablösung vom gewohnten Lebensumfeld einleitet und eine Phase der Status- und Rollenambivalenz darstellt. Wie die Pilgerreise ist die Migrationsreise ein außeralltägliches Unternehmen und wird als ein solches empfunden. ${ }^{1}$ Aufgrund des bis zuletzt ungewissen Ausgangs provoziert die Migrationsreise widersprüchliche Gefühle von Zuversicht und Angst, Hoffnung und Zweifel.

Die Reiseerfahrungen der Migrantinnen setze ich in Beziehung zu den strukturellen Bedingungen und dem Selbstbild der Subjekte (Goffman 1973: 127 ff.). Damit werden Erfahrungen und Bedeutungen im Moment der Statusveränderung zum „Immigranten“ thematisiert, eine Dimension internationaler Arbeitswanderungen, die eher selten ethnologisch bearbeitet wird, unter anderem deswegen, weil die Migrationsreise nur in Ausnahmefällen durch direkte Teilnahme erforscht werden kann. $^{2}$ Teilnehmende Beobachtung an Grenzposten ist politisch untersagt. Unter spanischen Juristen und Soziologen herrscht großes Nichtwissen darüber, was an den Grenzen, insbesondere am Madrider Flughafen Barajas, wirklich geschieht, wie viele südamerikanische Migranten Anfang bis Mitte der 2000er Jahre unmittelbar abgeschoben worden sind (Moya Malapeira 2006).

$1 \mathrm{Zu}$ ritualtheoretischen Interpretationen von Pilgerfahrten, siehe Peveling (2009); zu Pilgerfahrten bei Migrantinnen, siehe Liebelt (2010).

2 Einige wenige investigative Journalisten wagen es, sich mit ,illegalen“ Migranten auf die Reise zu begeben, siehe Gatti (2010). 
In meiner Untersuchung werden Begegnungen mit spanischen Beamten sowie Handlungen und Erfahrungen bolivianischer Migrantinnen nachträglich rekonstruiert und interpretiert. Die Analyse basiert auf einer Langzeitfeldstudie von achtzehn Monaten bei südamerikanischen Einwanderern in Sevilla sowie zwanzig narrativen Interviews mit bolivianischen Migrantinnen. Ich gehe davon aus, dass die restriktiven Bedingungen und schwierigen Lebensverhältnisse für Undokumentierte die Tendenz verstärken, die eigene Biographie in ein Vor und ein Nach der Auswanderung zu scheiden (vgl. Sayad 1999: 47 ff.). Außerdem wird das Bedürfnis, sich in einer schlüssigen Erzählung des eigenen Lebens wiederzufinden, durch die Migration erhöht. Der Sinn einer durch Rückschläge geprägten Migrantenexistenz muss immer wieder bestätigt werden. Das narrativ-biografische Interview ist in dieser Hinsicht ein ideales Format, um die Migrationsentscheidung vor sich selbst und anderen zu rechtfertigen, der eigenen Lebensgeschichte Bedeutung $\mathrm{zu}$ verleihen und Vorstellungen neu zu fassen. Es animiert die Gesprächspartner, „Kohärenzerzählungen“ (Bourdieu 2005; sh. Denzin 1989) zu formulieren. Diese wiederum geben Aufschluss über subjektive Verarbeitungsstrategien und kollektive Erfahrungsmuster.

\section{Südamerikanische und bolivianische Einwanderung nach Spanien}

Die meisten der rund zwei Millionen Südamerikaner, die sich aktuell in Spanien aufhalten, sind in den 1990er und 2000er Jahren eingereist (INE 1996, 2008). Spanien hatte in diesem Zeitraum mit das stärkste Bevölkerungswachstum durch Migration weltweit zu verzeichnen ${ }^{3}$; $40 \%$ der spanischen Neuzugänge zwischen 2000 und 2007 kamen aus Südamerika (López de Lera 2007: 4). Die Einwanderung verlief in Zyklen hohen Wachstums mit anschließender Konsolidierung: In den $1990 \mathrm{er}$ Jahren stieg zunächst die peruanische Einwanderung, bevor ab 1997 ecuadorianische und kolumbianische Zuzüge stark zunahmen. Im Jahr 2002 erlebte die seit längerem existente argentinische Immigration einen enormen Anstieg; ab 2004 schließlich begann die bolivianische Einwanderung. Bis dahin hatte sich die bolivianische Emigration auf Destinationen in Argentinien und den USA beschränkt, nun erfolgten Arbeitswanderungen in sämtliche spanische Städte und Regionen (Whitesell 2008). Von nicht einmal $7.000 \mathrm{im} \mathrm{Jahr} 2001$ stieg die Zahl der

3 Im Zeitraum von 1990 bis 2005 verzeichneten die USA (15 Millionen), Deutschland und Spanien (jeweils über vier Millionen Neuzugänge) das numerisch stärkste Bevölkerungswachstum durch Migration weltweit (UN General Assembly 2006: 39 ff.). 
bolivianischen Migranten in Spanien bis Anfang 2008 auf 240.000 (INE 2008). Die bolivianische Botschafterin in Madrid Carmen Almendras Camargo sprach Ende 2007 sogar von 300.000 bolivianischen Staatsbürgern, die sich in Spanien aufhielten (Fundación Ceimigra, Valencia, 30.11.07). Bis Anfang 2011 ist die Zahl auf 210.000 zurückgegangen (INE 2010), was auf die spanische Wirtschaftskrise zurückzuführen ist.

Wie die gesamte südamerikanische Einwanderung hat auch die bolivianische ein weibliches Profil. ${ }^{4}$ Der Frauenanteil der bolivianischen Bevölkerung in Spanien liegt bei rund $55 \%$; die Frauen sind zum großen Teil als Pioniermigrantinnen gekommen, das heißt, als eigentliche Arbeitsmigrantinnen vor den männlichen Familienangehörigen (Fouassier 2007: 4). Die interviewten bolivianischen Migrantinnen sind ohne Ausnahme vor ihren Partnern nach Spanien gereist, mehrheitlich in den Jahren 2005 und 2006. Viele besaßen nur einen losen Kontakt zu einem entfernten Verwandten. Alle zwanzig Befragten wohnten vor ihrer Emigration in der Stadt (vier im östlichen Santa Cruz de la Sierra, sechszehn im zentralen, zwischen Hochland und Tropen gelegenen Cochabamba) und kommen zum allergrößten Teil aus Land-StadtMigrantenfamilien, mehrheitlich aus dem indigenen Andenhochland. Die in der Stadt aufgewachsenen Frauen sprechen nur noch gebrochen Quechua. Spanisch war ihre Unterrichtssprache und wird auch in den Familien hauptsächlich benutzt. Auch die Kleidung ist bereits in Bolivien in den meisten Fällen westlich. Die Herkunftsfamilien gehören unabhängig von ethnischer und regionaler Herkunft zu den unteren städtischen Schichten in den durch Landbesetzung entstandenen Vorortsiedlungen von Cochabamba und Santa Cruz de la Sierra, haben jedoch über zwei Jahrzehnte eine Verbesserung ihrer Lebensverhältnisse erreichen können. Nur drei der interviewten Frauen sind den mestizischen Mittelschichten zuzuordnen; ein Verhältnis, das nach meinen Schätzungen die Zusammensetzung der bolivianischen Community in Sevilla annähernd repräsentiert.

Das Alter der Migrantinnen lag bei der Einreise zwischen 20 und 33 Jahren; knapp die Hälfte ließ einen Partner und Kinder in Bolivien zu-

4 Während die afrikanische und asiatische Immigration (außer Phillipinen) weiterhin von männlichen Arbeitssuchenden geprägt wird, sind die südamerikanischen und osteuropäischen Arbeitswanderungen nach Spanien typische Beispiele der internationalen Feminisierung der Migration (Han 2003). Bereits in den 1980er Jahren startete die Einwanderung von Frauen aus bestimmten Ländern Lateinamerikas und den Phillipinen. Die Nachfrage nach ausländischen Arbeitskräften für haushaltsnahe Dienste hat in Spanien seitdem weiter zugenommen; eine mangelhafte institutionelle Vermittlung von Angebot und Nachfrage fördert die Vergabe der Stellen an bereits im Land lebende irreguläre Migrantinnen (Colectivo IOE 2001; Herranz 1998, 2000; Zontini 2004; Aparicio Wilhelmi/Roig Molés 2006). 
rück. Die Partner reisten ohne Ausnahme bis April 2007 nach; Kinder wurden bisher keine nachgeholt. Es bestehen erhebliche Unterschiede zwischen den Befragten hinsichtlich Schulbesuch und Ausbildungschancen: Jeweils zu einem Drittel haben die Frauen in Bolivien nur die Grundschule besuchen können, mit mittlerer Reife abgeschlossen oder das Abitur gemacht. Von den im städtischen Umfeld sozialisierten Abiturientinnen hatten eine ein Studium und drei eine Berufsausbildung begonnen, sie jedoch aufgrund finanzieller Probleme und zugunsten der Migrationsentscheidung aufgegeben. Nur eine der Frauen war vor der Ausreise in einem Ausbildungsberuf als Krankenschwester tätig. Nahezu alle Migrantinnen haben neben Schule und Ausbildung in wechselnden Gelegenheitsjobs gearbeitet, in typischen prekären und feminisierten Bereichen des Kleinhandels und haushaltsnaher Dienstleistungen (als Wäscherin, Kellnerin, Tagesmutter, Straßenverkäuferin, im Geschäft der Eltern oder in der Telefonvermittlung).

In Sevilla sind die Interviewten wie die absolute Mehrheit aller lateinamerikanischen Migrantinnen in privaten Haushalten beschäftigt, entweder bei einer einzigen Familie als Ganztages-Kinder- bzw. Altenbetreuerin oder als Reinigungskraft und Tagesmutter auf Stundenbasis. Mit einer einzigen Ausnahme haben alle Frauen zunächst ohne Aufenthalts- und Arbeitsgenehmigung in Sevilla gelebt und gearbeitet. Bis Ende 2008 hatten nur vier von ihnen eine Statusnormalisierung in die Wege geleitet. ${ }^{5}$ Das entspricht den gesamtgesellschaftlichen Daten: In dem untersuchten Zeitraum verfügten nur etwa ein Viertel aller bolivianischen Migranten in Spanien über einen regulären Aufenthaltstitel (Fouassier 2007: 5 ff.; Ministerio de Trabajo e Inmigración 2007; INE 2008).

\section{Reisebedingungen und Erfahrungen bolivianischer Migrantinnen}

Der Zuzug aus Bolivien und anderen südamerikanischen Ländern begann unter relativ freizügigen Mobilitätsbedingungen, da für sämtliche Länder des Subkontinents Visumsfreiheit für Kurzaufenthalte bestand. Freizügigkeitsabkommen aus den 1960er Jahren, die das Franco-Regime mit iberoamerikanischen Ländern schloss, um Spaniern die

\footnotetext{
5 Nach der letzten Generalamnestie 2005 gibt es in Spanien noch individuelle Wege der Aufenthaltslegalisierung: nach drei Jahren nachgewiesenem Aufenthalt und einem aktuellen Arbeitsvertrag (arraigo social), nach einem zweijährigen kontinuierlichen Arbeitsverhältnis und seiner Verlängerung (arraigo laboral) oder über einen Arbeitsvertrag in den Sektoren des Arbeitsmarktes mit schwieriger Bedarfsdeckung (Aja 2006).
} 
Reise und Arbeitsaufnahme in Lateinamerika zu erleichtern, hatten einen begünstigenden Rechtsrahmen geschaffen. Die bilateralen Abkommen enthielten, für Ecuador gut dokumentiert, neben der Visumsfreiheit die Niederlassungsfreiheit, das Recht auf freie Berufswahl und die Inländergleichbehandlung (Carillo Espinosa et al. 2007). Mit den Einwanderungswellen wurden sie von spanischer Seite jedoch aufgekündigt. Die sukzessive Einführung des Schengen-Visums ${ }^{6}$ für Menschen aus Kuba und der Dominikanischen Republik, dann aus Peru, Kolumbien und Ecuador und schließlich Bolivien hat die postkoloniale Mobilität im iberoamerikanischen Raum unter dem Harmonisierungsdruck der EU-Kommission und dem Drängen der europäischen Staatschefs beendet (vgl. Spieß 2007: 17 ff.).

\section{Politisch-ökonomische Bedingungen}

Nach EU-Recht waren bolivianische Staatsbürger bis zur Einführung des Schengenvisums am 1. April 2007 berechtigt, mit nicht mehr als einem gültigen Pass nach Spanien zu reisen. Faktisch jedoch wurden bereits vor dem Stichtag darüber hinausgehende Dokumente gefordert (Devisen, Rechtfertigung des Aufenthalts, Rückreisegarantien). Das machte den Grenzübertritt zu einem Vabanquespiel. Aufgrund der Unwissenheit darüber, was genau gefordert wurde, standen und fielen die bolivianischen Migrationsvorhaben mit den Informationen und Angeboten der sogenannten Migrationsindustrie. ${ }^{7}$ Die Akteure der Migrationsindustrie, zu denen auch Tourismusunternehmen gehören, haben immer ein Interesse daran, Migrationsbewegungen zu stärken und ihre Routen an veränderte Rahmenbedingungen anzupassen. Je undurchsichtiger Politik und Rechtsprechung, desto effizienter können sie wichtige Informationen privatisieren. Reisebüros beispielsweise

${ }^{6}$ Eine der ersten Maßnahmen des Schengener Übereinkommens war die Angleichung der Einreisebestimmungen für Kurzaufenthalte bis zu drei Monaten. Das Schengen-Visum ist das einzige dem gemeinschaftlichen Recht unterliegende Visum. Zuletzt wurde 2002 auf dem EU-Ratsgipfel in Sevilla angemahnt, nunmehr die Liste der visumspflichtigen Drittländer kontinuierlich zu überprüfen. Im Jahr 2007 standen mit 133 Staaten so viele Länder wie nie zuvor auf der Liste, 1993 war man mit 73 Ländern gestartet (Lahav 2000: 232; Fouassier 2007; vgl. Wihtol de Wenden 2000: Kap. 3).

7 Zur „Migrationsindustrie“ gehören Reisebüros, Transportunternehmen und alle Arten von Arbeitsrekrutierern und Vermittlern aus den Herkunftsländern (Kirchenführer, Lehrer und andere einflussreiche Personen, zum Teil auch Leute, die selbst Migranten sind oder waren), aber auch Rechtsanwälte, Banken, Immobilienhändler und Dolmetscher im Ankunftsland. Auch kriminelle Schlepperbanden werden hinzugezählt. Migrationsindustrien entwickeln sich parallel zu sich verdichtenden transnationalen Netzwerken, die jeden Migrationsprozess kennzeichnen (Castles / Miller 1998: 97 f., Kap. 2; Pedone 2003: Kap. V). 
profitieren von den sich ständig verschärfenden Einreisevorschriften für EU-Drittstaatler, weil sie immer teurere Pauschalreisen verkaufen können, die eine Rückkehr der Migranten simulieren sollen. Durch eine Schengenrichtlinie von 2000 ist das Interesse, die Abschiebewahrscheinlichkeit der Klienten zu minimieren, sogar noch gestärkt worden. Neben dem zu befürchtenden Imageverlust bei Rückführungen und einem höheren Gewinn durch teure Reisepakete müssen nun sämtliche Kosten der Rückführung von den Fluglinien, Reiseagenturen und Veranstaltern getragen werden (Moya Malapeira 2006).

Im untersuchten Fall haben Reisebüros in den Städten Cochabamba und Santa Cruz de la Sierra Strategien des Eintritts in den Schengenraum entwickelt. Aus den Interviews mit den Migrantinnen geht hervor, dass die Reiseagenturen verschiedene Anreizsysteme nutzten, um Klienten zu gewinnen und sie davon zu überzeugen, dass die Einreise nach Spanien erfolgreich sein würde. Die Agenturen verfügten über ein preislich abgestuftes Flug- und Reiseangebot nach Erfolgswahrscheinlichkeit (vgl. Allgäuer 2009: 119). Zu den Standardofferten, von den Interviewten immer wieder genannt, gehörten Reisepakete mit Hin- und Rückflug, Reisekrankenversicherung sowie Reservierungen in Vierund Fünf-Sterne-Hotels für die ersten Tage. Außerdem wurde den Reisenden nahegelegt, 1000 bis 1500 \$ oder eine Kreditkarte mit sich zu führen. Sie wurden auf typische Fragen der Grenzbeamten vorbereitet, ihnen wurde eine bestimmte Haltung empfohlen und audio-visuelles Material gezeigt, dass ihnen die räumliche Orientierung am Ankunftsflughafen erleichtern sollte. Auch bestimmte Routen wurden von den Unternehmen vorgeschlagen.

Die Reiseagenturen reagierten damit auf Abschiebungen ihrer Passagiere. Bereits eine Polizeiverordnung von 2001 hatte unmittelbare Rückführungen kolumbianischer und ecuadorianischer Passagiere vom Madrider Flughafen zur Folge gehabt (Pedone 2003: 251 ff.). Durch eine im selben Jahr folgende Änderung des spanischen Ausländergesetzes (nun wurden alle Menschen mit der bloßen Absicht eines Grenzübertritts aus illegitimen Gründen dem Schnellverfahren der Abschiebung (devolución) ausgesetzt) mussten nun nicht mehr nur der Übertritt selbst legitim sein, sondern alle Aufenthaltspläne (Moya Malapeira 2006; Aja 2006). Diese Norm und weitere Zusatzbestimmungen und Revisionsverordnungen haben den individuellen Ermessensspielraum der Grenzbeamten erhöht. Dieser auch als behördliche Willkür zu beschreibende Spielraum, ein vom Franco-Regime geerbtes Problem spanischer Rechtsstaatlichkeit ${ }^{8}$, legt die Vermutung nahe, dass der direkte

8 Rechtsstaatliche Lücken und behördlicher Ermessensspielraum im Umgang mit Immigranten, ein Erbe des Franquismus, müssen durch einfache Gesetze geschlossen werden, da die gültige Verfassung von 1978 die spanische Emigration, 
Interaktionsprozess zwischen Beamten und Reisenden zu einem Mittel ihrer „Echtheitsbestimmung“ wurde und die Beamten eine letztendlich subjektive Scheidung (vermutlich nach quantitativen Vorgaben) in legitime Touristen und illegitime Arbeitsmigranten vornahmen. ${ }^{9}$

Auf dem Höhepunkt der bolivianischen Einwanderung zwischen Mitte 2006 und März 2007 berichteten bolivianische Medien über massive Abschiebungen bolivianischer Reisender in Barajas: „Einige fünfzig am Tag“ und „die Hälfte eines Fluges“ waren die Schlagzeilen (Whitesell 2008: 294). Auch die Einheitsgewerkschaft der Nationalen Polizei Spaniens erklärte im Winter 2006/2007, dass monatlich 1.500 bolivianischen Staatsbürgern der Zugang zum spanischen Hoheitsgebiet verwehrt würde, obwohl ihre Dokumente und Sicherheiten den formellen Anforderungen entsprächen (vgl. Hinojosa 2009: 82).

Die interviewten Bolivianerinnen erlebten bereits vor Mitte 2006 Fangfragen und Kriterienwillkür in einer Weise, die der spanische Oberste Gerichtshof Ende November 2007 als nicht rechtskonform einstufen sollte. Die Richter argumentierten, dass eine Reiseroutenplanung und die Vorbereitung auf Sehenswürdigkeiten keine Bedingungen für die Einreise sein dürfen und Abschiebungen nicht mit mangelndem Wissen über Spanien gerechtfertigt werden können. Den Tausenden abgewiesenen Bolivianern nützte dieser späte Entscheid nicht mehr. Zwischen 2005 und 2007, als die große Mehrheit der bolivianischen Reisenden alle formalen und finanziellen Anforderungen der Einreise erfüllten, reichten mangelhaftes Wissen oder eine labile Psyche, um als Migrant überführt zu werden:

„Viele wurden zurückgeschickt. Einem Freund wollte der Grenzpolizist eine Falle stellen. Er pochte darauf, dass er ihm die Wahrheit sage, auch wenn er zum Arbeiten gekommen sei, er würde ihn durchlassen, aber die Wahrheit müsse

aber kaum die Immigration thematisiert. Das Gesetz 4/2000 sollte den Ermessensspielraum der Beamten einschränken, bereits 1987 hatte der Oberste Gerichtshof die administrative Willkür (arbitrariedad administrativa) bei der Behandlung undokumentierter Immigranten kritisiert. Das reformierte 4/2000 jedoch, als LO 8/2000 in Kraft getreten und 2001 konkretisiert, beförderte erneut uneinheitliche Praktiken bei Festnahme, Freiheitsentzug und Abschiebung, insbesondere durch die oben genannte Klausel der unlauteren Motive der Einreise (Moya Malapeira 2006: 56 ff.; Aja 2006).

9 Die Abweisung südamerikanischer Reisender mit hinreichenden Reisedokumenten konnte zu diplomatischen Verstimmungen führen wie zu Beginn 2009, als Brasilien auf die wiederholte willkürliche Abweisung brasilianischer Bürger am Flughafen Barajas mit denselben Maßnahmen reagierte und Spaniern die Einreise verweigerte. Das aufstrebende Schwellenland konnte sich einen solchen öffentlichkeitswirksamen Schritt leisten, von Anfragen und Beschwerden bolivianischer oder ecuadorianischer Behörden ist nichts bekannt, falls es sie gab, haben sie weder Gehör noch Medienecho gefunden (vgl. Moya Malapeira 2006). 
er sagen (...). Andere Leute haben sie gefragt, wer der Präsident Spaniens oder der Autonomen Region sei, in die sie fahren wollten."

(Ninosca H. aus Cochabamba, 28 Jahre, Einreise im Frühjahr 2005)

\section{Einreiseoptionen und Taktiken der Migrantinnen}

Gerade weil Erfolg und Misserfolg der Einreise in starkem Maße von der direkten Interaktion mit den Beamten abhingen, verfügten bolivianische Reisende über einen gewissen Handlungsspielraum. Der Begriff des Handlungsspielraumes bezieht sich hier auf Verhandlungsspannen der von Verwaltungs- und Rechtsvorschriften betroffenen Bürger (Fiala 2007). ${ }^{10}$ Je stärker die behördliche Willkür, desto schlechter kann die Person die Konsequenzen ihrer Handlungen voraussehen, aber desto offener ist das Ergebnis. Ausländer stehen vor besonderen Schwierigkeiten, da sie zu Beginn weder mit dem Rechtssystem und der Bürokratie des Landes noch mit der Ausländergesetzgebung und ihrer Anwendung vertraut sind. Es ist davon auszugehen, dass bolivianische Reisende ohne die Vorbereitung einer bestimmten körperlichen Pose und einer verbalen Argumentationsweise weniger Chancen gehabt hätten.

Vor diesem Hintergrund erscheinen die Vorschriften und Empfehlungen der Reiseagenturen weniger unberechtigt und anekdotenhaft. Die bolivianischen Migrantinnen hatten sie für die Reise internalisiert. Sie verinnerlichten den ungefähren Aufbau der Ankunftshallen, Gepäckund Passabfertigungen, lernten im Flugzeug touristische Ziele und Fakten über Spanien auswendig, zeigten Entschlossenheit während der Unterredung mit Grenzpolizisten, führten nur ein Minimum an Gepäck, Kleidung und persönlichen Gegenständen mit sich und hatten sich Argumente und Rechtfertigungen ihrer vermeintlichen Ferienreise überlegt. Da es keine allgemeinverbindlichen Kriterien für Erfolg oder Misserfolg gab, erklären die Migrantinnen ihre eigene Erfolgsgeschichte nachträglich mit einer Mischung aus Glück und Zufall („Es ist wie eine Lotterie“) und der Stärke des persönlichen Auftritts an der Grenze. Entscheidend sei gewesen, trotz der Standardisierung des Verhal-

10 Die public policy analysis untersucht Verfahren und Praktiken der Implementierung von gesetzlichen Vorschriften auf verschiedenen administrativen Ebenen (siehe Pal 1997). Ein Strang dieses soziologischen Ansatzes arbeitet stärker empirisch-interaktionsdynamisch und studiert den direkten Kontakt zwischen Beamten und Bürgern (Fiala 2007). Siehe auch die Untersuchung von Sarah Lund (2001) über bürokratische Räume und das Prozedere zur Erlangung nationaler Ausweispapiere und die Erfahrungen indigener Personen mit der Staatsverwaltung in Cuzco, Peru. 
tens, individuelle Argumentationsweisen zu finden. Die folgenden Zitate zeigen, dass sich die Rechtfertigungsstrategien der Frauen nicht all$\mathrm{zu}$ weit vom eigenen Lebenshorizont entfernten.

Miguelina aus Cochabamba gelangte über den Pariser Flughafen Orly in den Schengenraum. Ihre Nacherzählung zeigt eine junge Frau, die den Grenzbeamten mit Charme und Enthusiasmus überzeugt, dass sie Frankreich besuchen will, und das, obwohl sie den Ehemann und vier kleine Kinder tags zuvor in Cochabamba zurücklassen musste:

In Paris fragten sie mich etwas auf Französisch. Ich antwortete, ,ich möchte Dein Land sehen und mich amüsieren' und hob voller Begeisterung die Arme. Sie werden nichts verstanden haben, wenn sie mich verstanden hätten (...).

(Miguelina H. aus Cochabamba, 29 Jahre, Einreise im August 2005)

Aus den Worten Miguelinas geht hervor, dass sie im entscheidenden Moment in der Lage war, die Rolle einer Touristin zu simulieren - wenn auch fast als Parodie. Sie sei, resümiert sie, auch tatsächlich neugierig gewesen, etwas von Paris zu sehen und war dementsprechend empört, als ihr Touristenführer bzw. ,Migrationsbroker' bereits am Flughafen verschwand (,Wir hatten einen Führer (guia), aber der hat uns gleich verlassen, er hat uns nichts gezeigt. Trotzdem habe ich Paris kennengelernt, ich mag Frankreich. “). ${ }^{11}$ Ihre Reisegruppe blieb zwei Nächte im für sie reservierten Hotel und fuhr dann mit dem Bus weiter nach Spanien.

Bei einem Pariser Kaufhausbesuch, so Miguelina, habe sie das Gefühl gehabt, dass alle Leute sie auf der Rolltreppe anstarrten und dachten, dass „diese Bolivianer sie bestehlen wollten“. Diese Wahrnehmung ist nicht vornehmlich als Unrechtsbewusstsein angesichts der Verschleierung ihrer Intentionen zu interpretieren ${ }^{12}$, sondern hat mit Stereotypen und Diskriminierungen zu tun, denen sie als Migrantin mit indigenbäuerlicher Herkunft in Cochabamba ausgesetzt gewesen ist und auf den neuen Kontext übertrug. Obwohl Miguelina von Momenten spricht, die den europäisch-bürgerlichen Idealvorstellungen einer Reise nahe-

11 Vergleichsinterviews mit männlichen Bolivianern und ecuadorischen Migrantinnen und Migranten zeigen, dass es verbreitet gewesen ist, zusätzlich zu den Hotelreservierungen Reisetouren zu buchen, meist durch Spanien. In diesen Fällen wurde die Gruppe tatsächlich von einem „Reiseführer“ begleitet; die Teilnehmerzahl nahm von Station zu Station ab, da die Migranten nacheinander ihre Zielorte erreichten.

12 Die legale Einreise als Tourist zwecks ,illegaler“ Arbeitssuche wird von Bolivianern seit Jahrzehnten in Argentinien betrieben. Sowohl in Argentinien als auch in Spanien sind in den letzten Jahrzehnten zahlreiche Amnestien für irregulär beschäftigte Ausländer durchgeführt worden. Auch nach Abschaffung dieser Option in Spanien sind die Migranten weiterhin der Meinung, dass Arbeit faktisch vorhanden ist und keine illegale Betätigung sein kann. 
kommen - die eigene Horizonterweiterung und Öffnung für neue Erfahrungen (z. B. die ethnische Vielfalt von Paris: „Alles ist voll von Schwarzen, erst hatte ich Angst, aber es ist gar nichts passiert.") - zeigt die Kaufhaus-Anekdote, dass sie die Rolle der unbekümmerten Touristin nicht aufrechterhalten konnte. Das Selbstbild als „arme“ Bolivianerin verweist auf eine fast schizophrene Rollensituation, sich als wohlhabende Urlaubsreisende ausgeben zu müssen, obwohl man sich der eigenen untergeordneten Position mehr als bewusst ist.

Auch Gabriela P. ist über Cochabamba, Buenos Aires und Madrid in Paris gelandet. Sie ist 24 Jahre alt und ließ den Partner und einen zweijährigen Sohn zurück. Gabriela stammt ebenfalls aus einer Familie von Hochlandmigranten in Cochabamba und ist so zierlich, dass sie sich als naives Schulmädchen ausgeben konnte:

Ich bin über Frankreich gekommen und zum Glück haben sie mich nichts gefragt, nur, ob ich Urlaub machen würde. Ehrlich gesagt, bei meinem Kleinmädchengesicht, wie sollten sie da denken, dass ich zum Arbeiten komme. Es war zwei Tage nach meinem Geburtstag und so sagte ich, die Reise sei ein Geschenk.

(Gabriela P. aus Cochabamba, 24 Jahre, Einreise im März 2006)

Mit einer reinen Urlaubsreise zu argumentieren war angesichts der notorisch schlechten Wirtschaftslage Boliviens und der Schwäche des bolivianischen Peso jedoch heikel. Das große Risiko, an der Grenze zurückgeschickt zu werden, versinnbildlicht sich in ihrem Verständnis von „Migration“. Migrieren hieß für Gabriela und andere Bolivianerinnen, die „Migration“, sprich Migrationsbehörde oder Grenzkontrolle zu passieren ${ }^{13}$ :

Andere wurden gefragt, wie sie sich den Luxus eines Urlaubs leisten könnten, wo ihr Land doch das ärmste überhaupt sei. Das war das Schwierigste: pasar las migraciones.

(Gabriela P., siehe oben)

Am gängigsten war die Rechtfertigungsstrategie, jemanden besuchen zu wollen. Dabei wurden auch sehr entfernte Bekannte und Verwandte genutzt. Trotz Einladungsschreiben, einem absoluten Muss, wurden die Migrantinnen Details über die einladende Person gefragt. Diejenigen Frauen, die wie Alicia $\mathrm{H}$. bei der Einreise noch ungebunden und kinderlos waren, konnten außerdem angeben, dass sie Studentinnen seien und die Eltern alles bezahlten, was den Anschein wirtschaftlichen Wohlstandes erweckte:

13 Obwohl migrante keine gängige Eigenbeschreibung war, besaß der Begriff dennoch positive Konnotationen der Realisierung des geplanten Projekts und wurde erst später durch den in Spanien allgegenwärtigen und negativ konnotierten Begriff des inmigrante überlagert. 
Mich fragten sie, warum ich gekommen sei, ob ich die Person des Einladungsschreibens kennen würde, wie sie aussehe, ob ich eine Kreditkarte hätte. Ich antwortete, dass ich Studentin sei und meine Mutter alles bezahlte. (...) Du musstest mindestens 1000 dabei haben, ungefähr 60 bis $80 \$$ pro Reisetag. Aber eigentlich gab es kein Kriterium, um dich abzuweisen, es war wie eine Lotterie. Du hast von Anfang an gespielt, denn womöglich hattest du bei einer Reiseagentur in Bolivien einen Flug reserviert und wenn du wenige Tage später zum Kaufen gingst, war der Preis gestiegen.

(Alicia H. aus Cochabamba, 22 Jahre, Einreise im Sommer 2005)

Faktisch hatte Alicia ein Jahr lang Buchhaltung gelernt, nach der Ausbildung wollte sie zur Polizei gehen. Als ihr unverhofft ein Einladungsschreiben zukam, ursprünglich für eine Cousine gedacht, brach sie die Ausbildung ab und ging nach Spanien, um ihrer Mutter und den drei kleinen Geschwistern mit Rücküberweisungen zu helfen.

Dieses Ergreifen einer unverhofften Gelegenheit, die schnelle Entscheidungsfindung und der rasche Kauf von Flugtickets und Rundreisen innerhalb weniger Tage veranschaulichen die Not und Tugend einer „taktischen“ Handlungslogik (De Certeau 2000). Auch andere Frauen haben Schule und Hochschule noch kurz vor dem Examen für die Migration abgebrochen, was nicht nur wirtschaftliche Zwänge und die Anfälligkeit ihrer Berufspläne offenbart, sondern auch dem Praxissinn entspricht, dass eine Option nicht auf später aufgeschoben werden kann, wenn theoretisch der bessere Zeitpunkt wäre: Man ergreift oder verliert sie. Leute, die taktisch handeln müssen, haben nach Michel de Certeau keinen Rückzugsort, von dem aus „die Zeit zu besiegen wäre“. Stattdessen sind sie abhängig von der Zeit, abhängig von den Möglichkeiten, die sich bieten. Nichts, was sie tun, hat eine Garantie auf Dauer. ${ }^{14}$ Die bolivianischen Migrantinnen hingen von den sich kontinuierlich verschlechternden staatlichen und internationalen Rahmenbedingungen ab, sie konnten den Zeitpunkt der Auswanderung nicht selbst bestimmen.

Dass Alicia, Gabriela, Miguelina und die anderen Frauen offiziell als Touristinnen nach Spanien gekommen sind, spielt juristisch später keine Rolle mehr. Das spanische Ausländergesetz macht keinen Unterschied zwischen einmal regulär im Land gewesenen und von Anfang an undokumentierten Migranten. Es gab im Jahr 2000 den Versuch, Südamerikaner bei der Aufenthaltsnormalisierung zu bevorzugen und so-

14 Strategisches Handeln hingegen ist nach De Certeau den dominanten politischen, wirtschaftlichen und wissenschaftlichen Institutionen vorbehalten, die sich von ihrer Umwelt isolieren können. Ihre distanzierte und totalisierende Sichtweise stellt einen Sieg des Ortes über die Zeit dar, während subalterne soziale Gruppen an ihre taktischen Alltagshandlungen gebunden sind: „sie hängen von der Zeit ab, ohne sie zu beherrschen" (De Certeau 2000: LI ff.). 
mit anzuerkennen, dass sie im Unterschied zu anderen Herkunftsgruppen das EU-Grenzregime nicht verletzt hatten. Der Vorschlag wurde jedoch von der konservativen Regierung des Partido Popular gestoppt und von den Sozialdemokraten der PSOE nicht wieder aufgenommen (Moya Malapeira 2006).

\section{Emotionale Erfahrungen und Bedeutungen der Reise}

Der Reiseweg der Bolivianerinnen wird von einer emotionalen Dissonanz (Hochschild 1979: 564 f.) zwischen den Rahmenvorschriften einer Urlaubsreise und dem geprägt, was die Migrantinnen tatsächlich empfinden. Die gesamte Reise ist widersprüchlich, es überlappen sich Unsicherheit und Zuversicht, Trauer und Euphorie. Die Rechtsunsicherheit und Unberechenbarkeit der Situation an der Grenze rufen Ängste hervor. Dieser erste Kontakt mit spanischen Behörden, die Befragung, das Durchsuchen der Koffer und womöglich das Warten in separierten Zimmern vermitteln das Gefühl von Unrechtmäßigkeit. Die Reisenden werden in eine gewollte Defensivhaltung gebracht, in der sie ihren Zugang zum spanischen Territorium rechtfertigen müssen, obwohl alle Ausweispapiere den Vorschriften entsprechen.

Auch die Reisebüros schürten mit ihren Instruktionen die allgemeine Unsicherheit. Den Migranten wurde Härte und Ausdruckslosigkeit empfohlen, da sie keinerlei Schwäche zeigen dürften:

Da wir Touristen waren und Gefahr liefen, zurückgeschickt zu werden, sollten wir mit niemandem sprechen, nicht einmal unter uns, auch keinerlei Angst zeigen, nicht zittern. Die Reiseagentur sagte, es gäbe Videokameras im Flugzeug und bei dem kleinsten Verdachtmoment würden sie uns in einem anderen Flieger zurückschicken.

(Angélica C. aus Cochabamba, 32 Jahre, Einreise im September 2005)

Durch die starken Emotionen bohrte sich der Tag der Ankunft ins Gedächtnis vieler Frauen ein. Diese mussten auf meine Frage, seit wann sie in Spanien seien, einen Moment nachdenken und kalkulieren, in welchem Jahr sie gekommen waren. Den Tag jedoch nannten sie ohne zu zögern: „Ich kam an einem 10. Dezember an“; „Die zwölf Stunden im Flugzeug sind schwer zu vergessen, dieser Tag ist wie vorgestern".

Für einige der Frauen sind die Umstände der Reise und die ersten Erfahrungen in Spanien so widersprüchlich, dass ihre „Doppelrollen“Reise identitätsverwirrende Züge annimmt. Ein Beispiel hierfür ist Inés G., die aus Cochabamba kommt und nach Stationen in Santa Cruz de la Sierra, Sao Paulo und Paris in Madrid Barajas landet. Sie hat zwei 
Telefonnummern bei sich, die einer entfernten Bekannten in Murcia, wie sie Arbeitsmigrantin, und die Nummer eines Luxushotels an der Madrider Gran Vía. Inés beschreibt ihre anfängliche Verwirrung, die Euphorie und gleichzeitige Einsamkeit im exklusiven Hotelzimmer an Spaniens Broadway. Ihre Gefühle und Eindrücke werden vom unsicheren rechtlichen Status und dem Bewusstsein ihrer physischen Auffälligkeit geprägt:

Niemand hat mich abgeholt. Eine Stunde habe ich auf meinen Koffer gewartet, dann wusste ich nicht, welchen Ausgang ich nehmen sollte, ich war verzweifelt, mein Gehirn vernebelt. Ich rief das Hotel an, aber mein Selbstvertrauen war im Keller und ich wusste nicht, was ich fragen sollte. Ich dachte, dass sie vielleicht die Polizei anrufen würden. Das klingt dumm aus heutiger Sicht, aber in dem Moment denkst du nicht, dass du Tourist bist, du denkst, dass du illegal bist und sie dich jetzt sofort deportieren werden. Du denkst, dass du unbedingt so schnell wie möglich vom Flughafen wegkommen musst. (...) Ich bin dann mehrmals die Gran Vía hoch und runter gegangen, bis ich das Hotel endlich fand. Auf dem Zimmer bin ich erschöpft aufs Bett gefallen und eingeschlafen, ich hatte zuvor noch nie in einem Hotel übernachtet. Mein Zimmer hatte einen Blick, es war ein Traum, ich habe lange geduscht. Ich hatte zwei Betten - es war zur gleichen Zeit eine große Freude und Einsamkeit, da ich Familie und Beruf hatte zurücklassen müssen. In dem Moment konnte ich mir natürlich nicht vorstellen, wie die Arbeit sein würde. Ich fuhr erst einen Tag später nach Murcia, weil ich noch ein bisschen in Madrid bleiben wollte, um das alles auszunutzen.

(Inés G. aus Cochabamba, 25 Jahre, Einreise im März 2006)

Wie Miguelina H. hat Inés G. das Gefühl, als „,arme Bolivianerin“ und „Illegale“ wahrgenommen zu werden, außerdem vermisst sie ihre Familie. Gleichzeitig jedoch genießt sie den Hotelaufenthalt und die Stadt. Zwei Tage lang hat sie sich trotz aller Ambivalenzen annähernd wie eine Touristin gefühlt und verhalten. Im Nachhinein klafft eine umso größere Lücke zwischen diesem Eindruck - dem des Komforts und Wohlstandes - und den späteren Arbeitsbedingungen.

Während der Reise müssen die Frauen mit widersprüchlichen Fremdund Eigenzuschreibungen umgehen. Sie erleben eine ambivalente Phase der Statusveränderung und des Schwankens zwischen unterschiedlichen Rechtsdefinitionen ihrer Person. Über den gesamten Zeitraum der Reise hinweg sind sie offiziell Touristinnen, die jedoch unter dem Generalverdacht der irregulären Arbeitswanderung stehen, was sich im einschüchternden Grenzinterview materialisiert. Dem Selbstverständnis nach fehlt ihnen nicht nur ein politisch-rechtlicher Status, sondern auch eine soziale Rolle. Sie fühlen sich abgesehen von wenigen Momenten nicht als Touristinnen und dem Selbstbild nach sind sie noch keine wirklichen Migrantinnen, dafür müssen sie erst die migraciones passieren. Auch nach dem Grenzübertritt fehlt ihnen eine soziale Rolle, die sie erst mit der Aufnahme einer Beschäftigung erhalten. Als trabaja- 
dora migrante werden viele von ihnen später zu Haupternährerinnen ihrer transnationalen Haushalte. Damit wäre die Phase des Übergangs überwunden, der Moment der Statusveränderung zum Arbeitsmigranten genommen und eine neue Rolle im transnationalen Familienverband gefunden.

Victor Turners Ansatz, die Analyse ritueller Prozesse auch in modernen Gesellschaften fortzusetzen, lässt sich am Beispiel globaler Arbeitsmigrationen weiterdenken. Bekanntlich hat Turner das klassische Dreistufenmodell der Ablösung, Liminalität und Wiedereingliederung von Arnold Van Gennep vom engen Kontext einfacher Gesellschaften gelöst und in seinem Spätwerk weniger sozial-strukturell als vielmehr erfahrungstheoretisch gedeutet (Turner 1982; 1986). Migration als rituellen Prozess und die Migrationsreise als liminale Phase zu verstehen, meint, einen Analyserahmen für die Erfahrungsebene irregulärer Migration zu formulieren. Es möchte aktuelle Einreisebemühungen in die westliche Welt aus Sicht der Migranten darstellen und sie als das begreifen, was sie für Personen ohne Visum bedeuten: einen Weg mit ungewissem Ausgang, eine Lotterie mit ernsthaften Folgen, eine Reise des Risikos und der Gefahr, auf der eine zugleich politische, physische und biographische Grenze zu überwinden ist.

\section{Schlussbetrachtungen}

In den letzten zwei Jahrzehnten ist Spanien weltweit eines der wichtigsten Zielländer internationaler Arbeitswanderungen gewesen. Südamerikaner sind mehrheitlich als Touristen eingereist, so auch die meisten bolivianischen Migranten. Die interviewten bolivianischen Frauen erlebten die sukzessive Abschaffung eines Raums der Freizügigkeit für Iberoamerikaner am eigenen Leib. Ihre Migrationszeit war begrenzt. Die raschen Reaktionen auf momentan aufblitzende Mobilitätsoptionen zeigen eine „taktische“ Handlungslogik, die im Unterschied zu strategischem Handeln keine Macht über den Raum besitzt (De Certeau 2000). Die Überwindung von Raum und die Partizipation an globalen Mobilitätsmustern hängen von der sozio-ökonomischen und geographischen Position ab, von der man startet, sowie von Nationalität und Staatsangehörigkeit. In der Weltgesellschaft bilden sich neue, multiple "globalization frontiers" heraus (Halbmayer/Mader 2004); Visavorschriften produzieren eine solche Globalisierungsgrenze. Das hier skizzierte Beispiel zeigt jedoch auch, dass Menschen sich den Politiken der Immobilität durch Wege am Rande der Legalität erfolgreich widersetzen. Allerdings spielen hierbei Reiseunternehmen und andere Experten der „Migrationsindustrie“ eine wichtige Rolle, die das 
Wissen um Einwanderungsbestimmungen, Schlupflöcher und Reiserouten privatisieren und teuer verkaufen.

Der Interaktionsprozess zwischen Grenzpolizisten und bolivianischen Migrantinnen an den Ankunftsflughäfen war weit mehr als eine routinierte Ausweiskontrolle. Bei der Identifizierung ging es nicht nur darum, die Lückenlosigkeit und Kohärenz aller persönlichen Angaben $\mathrm{zu}$ prüfen und zu verfizieren, dass die Zeichen individueller Identität wie Foto, Fingerabdruck und Unterschrift tatsächlich auf die physische Person der Reisenden verwiesen. Über die Echtheitsbestimmung der Ausweispapiere hinaus wurde durch Interviews versucht, eine „Echtheitsbestimmung“ der Reiseintentionen vorzunehmen. Neben der Überprüfung materieller Dinge - Geld, Tickets, Hotelreservierungen, physische Identitätsmerkmale - mussten die Bolivianerinnen Fragen beantworten, die dem alleinigen Ziel galten, sie als „Undercover“Arbeitsmigrantinnen zu überführen. Diese Gewissens- und Wissensfragen unterlagen dem Ermessensspielraum einzelner Beamter. Nicht nur die Beurteilung der Antworten war selektiv, sondern auch die Auswahl der Fragen. Die Interviews waren eindeutige Exklusionsinstrumente (vgl. Hahn/Schorch 2007), die helfen sollten, Zutritt zu verweigern. Nicht nach objektiven Kriterien, sondern nach subjektivem Ermessen wurde über Menschen, ihre Zugangsrechte und Mobilitätschancen geurteilt.

Obwohl die Zahl der interviewten Personen nicht ausreicht, um repräsentativ zu sein, legt meine Studie doch nahe, dass die Frauen mit indigenem Phänotyp besondere Hürden zu überwinden hatten. Sie waren sich ihres Aussehens bewusst; die spanischen Grenzpolizisten wiederum mussten Kriterien finden, um eine bestimmte Anzahl von Passagieren zurückzuschicken. Wenige Aspekte sind so willkürlich, aber oberflächlich so evident wie die äußere Erscheinung. In den Worten Abdelmalek Sayads hatten die Migrantinnen an der Grenze „nichts als ihren Körper“, ihre Statur, ihren Namen und andere Marker, die sie in eine globale „visuelle Ordnung“ von arm und reich, indigen und europäisch setzte (Sayad 1999: 366). Da jedoch aus politisch-diplomatischen Gründen nicht alle bolivianischen Reisenden abgewiesen werden konnten, bestand ein gewisser Handlungsspielraum. Die interviewten Frauen konnten sich mit Taktiken der körperlichen und verbalen Kommunikation, mit demonstrativer Selbstsicherheit, Schlagfertigkeit und Wissen über Spanien für einen Moment „de-ethnisieren“: Einmal ist es paradoxerweise die physische Statur selbst, die als Vorteil ausgespielt wird; ein anderes Mal können die Intentionen durch Halbwahrheiten über die Berufsausbildung und das Studentenalter verschleiert werden; ein weiteres Mal wird Urlaubsfreude parodiert. 


\section{Literaturverzeichnis}

Aja, Eliseo (2006): Introducción. La evolución de la normativa sobre inmigración. In: Aja, E./Arango, J. (Hg), Veinte años de inmigración en España: perspectivas jurídica y sociológica (1985-2004), Barcelona (Fundació CIDOB).

Allgäuer, Alicia (2009): „Cholas, Churros, Chicharrón“: Prozesse der Ethnisierung und Vergeschlechtlichung in bolivianischen Migrationsgeschichten zwischen Cochabamba und Andalusien. Universität Wien (http://othes.univie.ac. at/4084, 11. 4. 2012).

Aparicio Wilhelmi, M./Roig Molés, E. (2006): La entrada por razones laborales y el trabajo de los extranjeros. El progresivo desarrollo de un sistema ordenado de entrada laboral. In: Aja, Eliseo y Joaquín Arango (Hg.), Veinte años de inmigración en España: perspectivas jurídica y sociológica (1985-2004), Barcelona (Fundació CIDOB).

Bourdieu, Pierre (2005): Was heißt sprechen? Zur Ökonomie des sprachlichen Tausches. Wien (Braumüller Universitäts-Verlagsbuchhandlung).

Carillo Espinosa, M. C. et al. (2007): Los estudios sobre la migración ecuatoriana: ¿Hacia una zona de prestigio en España? In: V. Congreso sobre la Inmigración en España. Valencia (Universität Valencia).

Castles, S./Miller, M. J. (1998): The Age of Migration: international population movements in the modern world. Basingstoke (Macmillan).

Colectivo IOE (2001): Mujer, inmigración y trabajo. Madrid (Ministerio de Trabajo y Asuntos Sociales).

De Certeau, Michel (2000): The practice of everyday life. Minneapolis: University of Minnesota Press.

Denzin, Norman K. (1989): Interpretive biography. Newbury Park (Sage).

Fiala, C. (2007): Más allá de las leyes: Prácticas de implementación de la política de inmigración en España. In: V. Congreso sobre la Inmigración en España. Valencia (Universität Valencia).

Fouassier, M. (2007): Inmigración boliviana en España y el País Vasco. In: V. Congreso sobre la Inmigración en España. Valencia (Universität Valencia).

Fundación Ceimigra (2007): Inmigración boliviana en Valencia. Valencia, unveröffentlichtes Tagungsdokument.

Gatti, Fabricio (2010): Bilal. Als Illegaler auf dem Weg nach Europa. München (Verlag Antje Kunstmann).

Goffman, Erving (1973): Asyle. Über die soziale Situation psychiatrischer Patienten und anderer Insassen. Frankfurt a. M. (Suhrkamp).

Hahn, Alois / Schorch, Marén (2007): Tests und andere Identifikationsverfahren als Exklusionsfaktoren. In: Gunsenheimer, Antje (Hg.), Grenzen. Differenzen. Übergänge. Spannungsfelder inter- und transkultureller Kommunikation, Bielefeld (transcript). 
Halbmayer, Ernst/Mader, Elke (2004): Kultur, Raum und Landschaft in Zeiten der Globalisierung. Zur Einleitung. In: Halbmayer, E./Mader, E. (Hg.), Kultur, Raum, Landschaft. Zur Bedeutung des Raumes in Zeiten der Globalisierung, Frankfurt a. M. (Brandes \& Apsel/Südwind).

Han, Petrus (2003): Feminisierung der Migration. Stuttgart (UTB).

Herranz Gómez, Yolanda (2000): Inmigración e incorporación laboral. In: Migraciones 8: $127-164$.

- (1998): La inmigración latinoamericana en diferentes contextos de recepción. In: Migraciones, 3: 31-51.

Hinojosa-Gordonava, Alfonso R. (2009): Burcando la Vida. Familias transnacionales en España. La Paz: PIEB/Editorial Presencia.

Hochschild, Arlie Russell (1979): Emotion Work, Feeling Rules, and Social Structure. In: The American Journal of Sociology, Vol. 85(3): 551-575.

Instituto Nacional de Estadística (INE) (1996, 2008, 2010): Explotación estadística del Padrón. Madrid. (http://www.ine.es, 30.07.2011).

Kopnina, H. (2007): Migration and Tourism: Formation of New Social Classes. New York (Cognitant Communication Corporation).

Lahav, Gallya (2000): The Rise of Nonstate Actors in Migration Regulation in the United States and Europe. Changing the Gatekeepers or Bringing Back the State? In: Foner, N./Rumbaut, R. G./Gold, S. J. (Hg.), Immigration research for a new century. Multidisciplinary perspectives, New Cork (Russel Sage Foundation).

Liebelt, Claudia (2010): Becoming Pilgrims in the „Holy Land“: On Filipina Domestic Workers' Struggles and Pigrimages for a Cause in Israel. In: The Asia Pacific Journal of Anthropology 11(3-4): 245-267.

López de Lera, Diego (2007): Incidencia de la inmigración latinoamericana en España. In: V. Congreso sobre la Inmigración en España. Valencia (Universität Valencia).

Lund, Sarah (2001): Bequeathing and quest. Processing personal identification papers in bureaucratic spaces (Cuzco, Peru). In: Social Anthropology, 9(1): 3 24.

Ministerio de Trabajo e Inmigración, Secretaria de Estado de Inmigración y Emigración (2008): Anuario Estadístico de Inmigración del año 2007 (http:// extranjeros.mtin.es / es / index.html, 05.05.2008).

Moya Malapeira, D. (2006): Evolución del sistema de control migratorio de entrada en España. In: Aja, Eliseo/Arango, Joaquín (Hg.), Veinte años de inmigración en España: perspectivas jurídica y sociológica (1985-2004), Barcelona (Fundació CIDOB).

Pal, Leslie A. (1997): Beyond Policy Analysis. Public issue management in turbulent times. Ontario (Nelson). 
Pedone, Claudia (2003): „Tú siempre jalas a los tuyos“: Cadenas y redes migratorias de las familias ecuatorianas hacia España. Universidad Autónoma de Barcelona (http://www.tdcat.cesca.es /, 15.03.2011).

Peveling, Barbara (2009): Tourismus der Rückkehr als kulturelle Reserve: jüdische Pilger aus Frankreich auf dem Weg nach Djerba und zurück. In: Zeitschrift für Ethnologie 134: 171-187.

Sayad, Abdelmalek (1999): La double absence: Des illusion de l'émigré aux souffrances de l' immigré. Paris (Seuil).

Spieß, Katharina (2007): Die Wanderarbeitnehmerkonvention der Vereinten Nationen. Ein Instument zur Stärkung der Rechte von Migrantinnen und Migranten in Deutschland. Berlin (Deutsches Institut für Menschenrechte).

Turner, Victor (1982): From ritual to theatre. The human seriousness of play. New York (Performing Arts Journal Publ.).

- (1986) (Hg.): The anthropology of experience. Urbana (University of Illinois Press).

UN General Assembly (2006): International migration and development. Report of the Secretary-General A/60/871 (http://www.un.org/esa/population/ migration/hld/index.html, 30.07.2011).

Whitesell, Lily (2008): Y aquellos que parieron: retratos del éxodo boliviano. In: Shultz, Jim/Crane Draper, M.: Desafiando la globalización. Historias de la experiencia boliviana. (http://www.democracyctr.org/publications / desafiando, 15.03.2011).

Wihtol de Wenden, C. (2000): ¿Hay que abrir las fronteras? Barcelona (Bellaterra).

Zontini, Elisabetta (2004): Immigrant women in Barcelona: coping with the consequences of transnational life. In: Journal of Ethnic and Migration Studies. Vol. 30, Nr. 6: 1113-1144.

\section{Summary}

This article deals with the journey of Bolivian migrant women to Spain. It analyses the structural conditions of the trip as tourists and „undercover" labour migrants and relates them to the womens' travel experiences. Important actors to avoid political restrictions of entry are travel agencies. It will be shown how the women situationally enact strategies to pass border controls and what they feel having to conceil their actual identity and intentions.

\section{Zusammenfassung}

Dieser Aufsatz beschäftigt sich mit der Reise bolivianischer Migrantinnen nach Spanien. Die politisch-ökonomischen Rahmenbedingungen der Reise als Touristinnen und „Undercover“-Arbeitsmigrantinnen werden analysiert und in 
Bezug gesetzt zu ihren Erfahrungen. Wichtige Akteure zur Umgehung migrationspolitischer Restriktionen sind Reisebüros. Es wird analysiert, welche Taktiken die Frauen verwenden, wie sie die Begegnung mit den Grenzpolizisten darstellen und was es emotional bedeutet, die eigene Identität zu verschleiern und Absichten zu verstecken.

\author{
Dr. Juliane Müller \\ Ludwig-Maximilians-Universität München \\ Institut für Ethnologie \\ Oettingenstr. 67 \\ 80538 München \\ E-Mail: eva_juliane_mueller@yahoo.de
}

